\title{
SuperHERO: Design of a New Hard-X-Ray Focusing Telescope
}

\author{
Jessica Gaskin, Ronald Elsner, Brian \\ Ramsey, Colleen Wilson-Hodge, \\ Allyn Tennant \\ NASA Marshall Space Flight Center \\ Huntsville, AL 35811 \\ 256-961-7818 \\ Jessica.Gaskin@nasa.gov
}

Paul Seller and Matthew Wilson Science and Technologies Facilities Council, Rutherford Appleton Laboratory, Harwell Campus, Oxfordshire, OX11 0QX, UK paul.seller@stfc.ac.uk
Steven Christe and Albert Shih NASA Goddard Space Flight Center 8800 Greenbelt Rd. Greenbelt, MD 20771 Steven.d.Christe@nasa.gov

David Stuchlik NASA Wallops Flight Facility Code 820, Wallops Island, VA 23337

david.w.stuchlik@nasa.gov

\author{
Kiranmayee Kilaru and \\ Douglas Swartz \\ Universities Space Research \\ Association, 320 Sparkman Dr., \\ Huntsville, AL 35805 \\ kiranmayee.kilaru-1@nasa.gov
}

\begin{abstract}
SuperHERO is a hard x-ray (20-75 keV) balloonborne telescope, currently in its proposal phase, that will utilize high angular-resolution grazing-incidence optics, coupled to novel CdTe multi-pixel, fine-pitch $(250 \mu \mathrm{m})$ detectors. The high-resolution electroformed-nickel, grazing-incidence optics were developed at MSFC, and the detectors were developed at the Rutherford Appleton Laboratory in the UK, and are being readied for flight at GSFC. SuperHERO will use two active pointing systems; one for carrying out astronomical observations and another for solar observations during the same flight. The telescope will reside on a light-weight, carboncomposite structure that will integrate the Wallops Arc Second Pointer into its frame, for arcsecond or better pointing. This configuration will allow for Long Duration Balloon flights that can last up to 4 weeks. This next generation design, which is based on the High Energy Replicated Optics (HERO) and HERO to Explore the Sun (HEROES) payloads, will be discussed, with emphasis on the core telescope components.
\end{abstract}

\section{TABLE OF CONTENTS}

1. INTRODUCTION .1

2. SUPERHERO CONCEPT ...................................2

3. X-RAY OPTICS...................................................5

4. COMPLIMENTARY DETECTORS.......................7

5. SYSTEM ALIGNMENT .....................................8

6. THE FUTURE .....................................................99

7. SUMMARY .......................................................11

ACKNOWLEDGEMENTS.....................................11

REFERENCES.................................................12

BIOGRAPHY .................................................14

\section{INTRODUCTION}

Focusing x-ray telescopes offer high angular resolution and high signal to noise through reduced background over nonfocusing $\mathrm{x}$-ray telescopes (i.e., coded mask apertures and mechanical collimators). This paper summarizes the design for a new high-resolution and spectroscopic Hard X-Ray (HXR) (20-75 keV) telescope, dubbed SuperHERO, suitable for use on a high-altitude balloon platform. Further application on a space-based platform is also discussed.

In the soft $\mathrm{x}$-ray regime (SXR), from $\sim 0.1$ to $\sim 10 \mathrm{keV}$, focusing $\mathrm{x}$-ray telescopes have made huge strides, culminating in the formation of the Chandra X-ray Observatory. Chandra offers sub-arcsec angular resolution and high-resolution dispersive spectroscopy [1]. Figure 1 shows the evolution in improved angular resolution for multiple missions: XMM-Newton, the Einstein Observatory, the Röntgen Satellite (ROSAT), and Chandra [2]. Half Power-Diameter (HPD) angular resolutions improve from 15 arcsecs for XMM-Newton to 0.6 arcsec for Chandra. The HPD is the standard metric for specifying (at a given energy) the angular diameter of the image of a point source that contains half the flux.

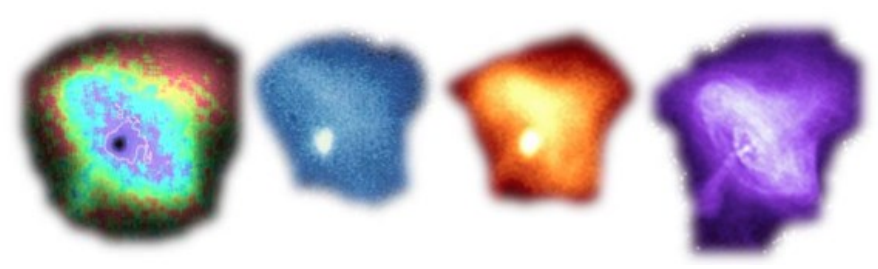

Figure 1. SXR images of the Crab Nebula taken with (from left to right) XMM-Newton, the Einstein Observatory, ROSAT, and the Chandra X-Ray Observatory. The HPD angular resolutions are 15arcsecs, 10 arcsecs, 5 arcsecs and 0.6 arcsec respectively. The Chandra image shows significantly more structure than in the other images.

It is clear from the figure that improved angular resolution offers additional insight into the structure and nature of an extended object. The high angular resolution afforded by focusing optics coupled to an appropriately matched highresolution detector is essential for mitigating source confusion in crowded fields, for direct imaging of extended sources on fine spatial scales and for efficient observing through greatly-increased sensitivity. 
In the HXR regime $(\sim 10 \mathrm{keV}$ to $\sim 120 \mathrm{keV})$, the NuSTAR telescope, for Nuclear Spectroscopic Telescope Array, is a NASA explorer-class mission that has illustrated the capability to make dramatic improvements in both sensitivity and angular resolution over previous HXR missions by utilizing grazing incidence optics coupled to matching focal plane detectors [3]. NuSTAR has recently made multiple discoveries that highlight the extreme importance of this energy region [4].

SuperHERO is a high-sensitivity, focusing HXR balloonborne telescope that will allow for significantly improved imaging resolution over that of any existing HXR telescope of astronomical objects and the Sun. Through collaborations between NASA Marshall Space Flight Center (MSFC), NASA Goddard Space Flight Center (GSFC), Rutherford Appleton Laboratory (RAL) and NASA Wallops Flight Facility (WFF), this telescope will mate the high resolution electroformed nickel-cobalt alloy mirror shells developed at MSFC with the fine $(250 \mu \mathrm{m}$ pitch) pixel CdTe detectors developed at RAL and tailored for flight at GSFC. The aim is to fly SuperHERO on a Long Duration Balloon (LDB) Flight from Antarctica, which will lead to the development of the next generation HXR telescope for space.

\section{SUPERHERO CONCEPT}

\section{HERO/HEROES Missions}

The basic design for SuperHERO originates with the High Energy Replicated Optics (HERO) telescope. HERO was a MSFC-developed balloon payload designed for astrophysical observations at sensitivities of a fewmilliCrab-level during a typical turnaround flight, in the 20$75 \mathrm{keV}$ HXR band. In 2001, HERO demonstrated the feasibility of flying high-resolution X-ray optics from a balloon platform when it captured the first focused HXR images from galactic sources using a prototype payload [5]. With further improvements in resolution of the X-ray optics and an increased sensitivity, HERO obtained the first focused image of the Crab Nebula at HXR energies of 20-60 $\mathrm{keV}$, revealing detail down to $\sim 26$ arcsec [6].

The High Energy Replicated Optics to Explore the Sun (HEROES) telescope used the HERO payload in its existing form to observe astronomical objects, but added a solar aspect system developed at GSFC [7] to allow for solar observations during the same flight. HEROES launched from Fort Sumner, NM in September of 2013, and successfully observed the Crab Nebula for three hours as well as an active region on the Sun for just over 7 hours [8].

Both HERO and HEROES were designed to fly a conventional balloon flight, which typically lasts no longer than $\sim 36$ hours.

\section{SuperHERO Overview}

SuperHERO is a next-generation payload with enhanced optics and new focal plane detectors on a gondola specifically designed for high-sensitivity measurements on LDB flights. This longer, 2 to 4 week, flight necessitates significant modifications to the design of the gondola, thermal and power management, and telemetry system, among other. The emphasis of this paper is on the development of the core payload (i.e., the HXR telescope) in the context of an LDB flight. A detailed description of the SuperHERO gondola and supporting instrumentation can be found in [9].

Gondola- For optimum LDB flight performance, SuperHERO must be significantly lighter than HERO/HEROES and have greatly improved pointing capability. To achieve this, the gondola and optical bench structures have been completely redesigned and the Wallops Arc-Second Pointer (WASP) pointing system (Figure 2) has been incorporated for arcsecond stability [10]. WASP, which is an inertial pointer that uses pitch-yaw articulated gimbals, has made 5 flights; the most recent with the HyperSpectral Imager for Climate Science (HySICS) and the Observatory for Planetary Investigations from the Stratosphere (OPIS). These latest flights flew from Fort Sumner, NM in August and October of 2014, respectively [11]. Table 1 summarizes key features and functionality associated with the SuperHERO gondola.

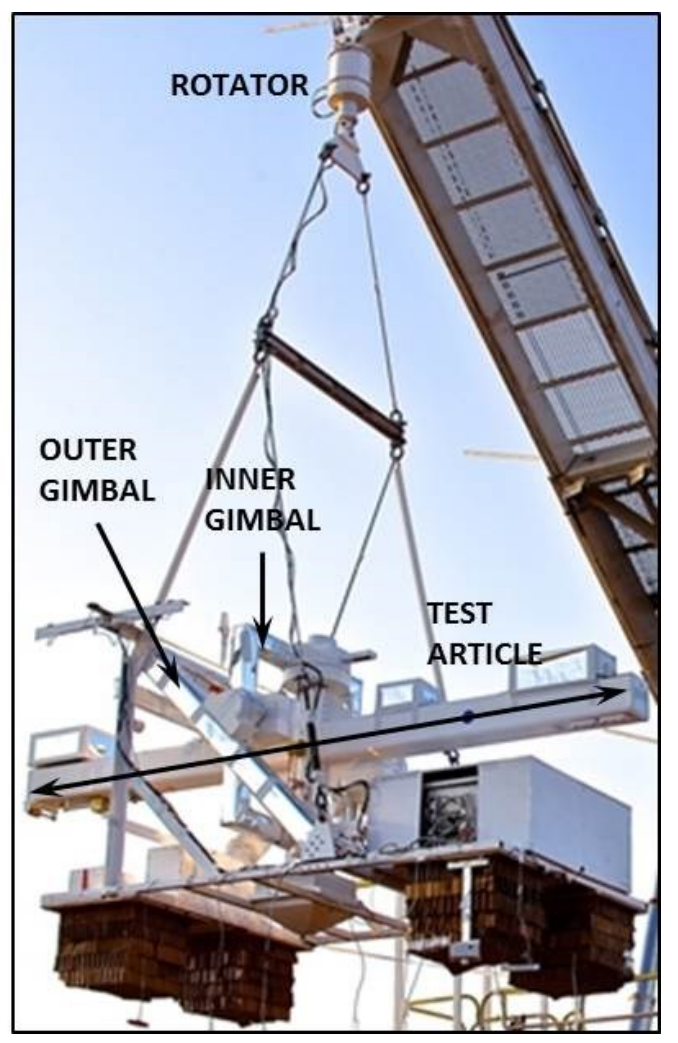

Figure 2. WASP test payload suspended from the launch vehicle in Fort Sumner, NM just prior to launch in 2012. WASP consists of an outer gimbal connected to a rotator via suspension cables. The inner gimbal supports the test article or primary payload. In this case, the primary payload is a 6-m-long bar. Image Credit: NASA 
Table 1. Summary of the SuperHERO gondola.

Key Component

Gondola Structure
Commercial Off The Shelf-Graphite fiber-epoxy tubing reinforced with sandwich panel plates with steel tubing center frame.

- Science Payload Weight: $<2,500$ lbs (estimated from heritage and modeling, includes core telescope)

- Power: Solar Panels + Batteries

\begin{tabular}{|c|c|}
\hline Pointing System & $\begin{array}{c}\text { Wallops Arc-Second Pointer (WASP) } \\
\qquad \quad \text { Pointing accuracy: } \sim 1 \text { arcsecond }\end{array}$ \\
\hline Rotator & $\begin{array}{l}\text { Provides coarse attitude control. } \\
\text { Payload Positioning: to within } \sim 1^{\circ} \text { of the desired azimuth when } \\
\text { combined with the GPS attitude determination unit [13]. }\end{array}$ \\
\hline Star Camera System & 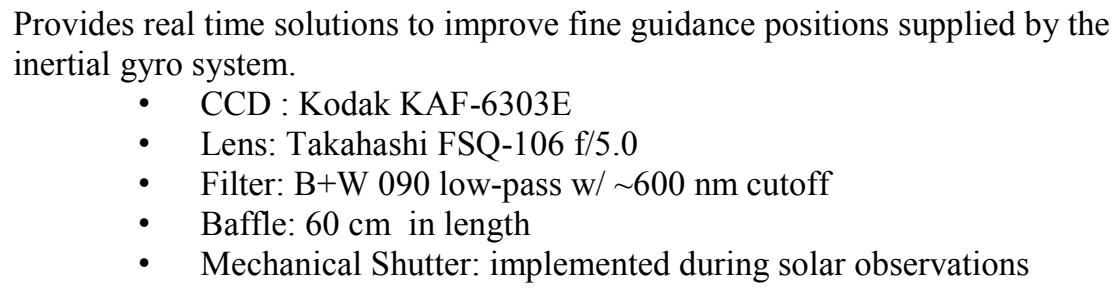 \\
\hline $\begin{array}{l}\text { Pitch-Yaw Aspect } \\
\text { System }\end{array}$ & $\begin{array}{l}\text { Part of the Solar Aspect System, provides } 2 \text { independent relative solar aspect } \\
\text { solutions and measures the alignment between optics and detectors. } \\
\text { - Aspect Solution: to } \sim 10 \text { arcsecs } \\
\text { - Optics-Detector Alignment: to } \sim 15 \text { arcsecs }\end{array}$ \\
\hline Roll Aspect System & $\begin{array}{l}\text { Measures roll during flight to reduce smearing of an x-ray source caused by } \\
\text { gondola pendulation and other transient tilts during post-processing. } \\
\qquad \quad \text { Roll Knowledge: }<0.3 \text { arcmin (relative) }\end{array}$ \\
\hline
\end{tabular}

The SuperHERO gondola frame is minimal, with lightweight structures for mounting the solar panels, ballast hoppers and an electronics platform (Figure 3). The upperstructure will be a tension cable system and rotator provided by NASA WFF. The gondola structure that attaches to the WASP and optical bench uses sandwich panel plates with a center frame of steel tubing welded to WASP. The sandwich plates are connected to one another using graphite fiber / epoxy wound tubes that have a near zero Coefficient of Thermal Expansion (CTE). The elevation flange has a steel frame as well, to distribute the loads from the vertical motion axis bearing attach points. The HERO/HEROES star camera, the HEROES Solar Aspect System (SAS) and much of the gondola electronics will be reused.

SuperHERO will have two aspect systems that will enable pointing at both astronomical objects and the Sun during the same flight. Astronomical pointing will be accomplished with the use of a star camera system combined with the gondola inertial gyro system [12]. The star camera will be mounted to the top of the sandwich plate to which the optics are also mounted. Solar pointing will be facilitated with the use of the SAS, which consists of a Pitch-Yaw Aspect System (PYAS) and a Roll Aspect System (RAS). The PYAS is made up of 2 independent systems, each of these provides relative solar aspect solutions that allow for a pointing knowledge of better than 10 arcsecs. These 2 independent systems also make a measure of the alignment between the optics and the detectors during solar pointing to an accuracy of around 15 arcsecs. The SAS is described in detail in [7]. On SuperHERO, the PYAS will be mounted down the center of the optical bench. The RAS will provide a precise measurement of the roll $(<18$ arcsecs). During flight, roll can be caused by the natural pendulation of the payload and other transients (high winds). During the solar measurements, it is critical to measure the roll so that any smearing of the x-ray source can be removed during post processing. The optimal RAS mounting location is yet to be determined.

Core Instrument - At the heart of SuperHERO exists the HXR telescope. This telescope consists of 152 highresolution HXR mirror shells, arranged into 8 modules. Each of these modules is aligned to a $2 \times 2$ array of novel fine-pixel $(250 \mu \mathrm{m}$ pitch $) \mathrm{CdTe}$ detectors, at a focal length of 6-m. The optics and detectors are mounted on an opentruss optical bench with low CTE. Similar open-truss structures have been used with good results [14]. The benefit is a lighter payload, without the loss of structural integrity. Both of these aspects are essential for a LDB flight. 


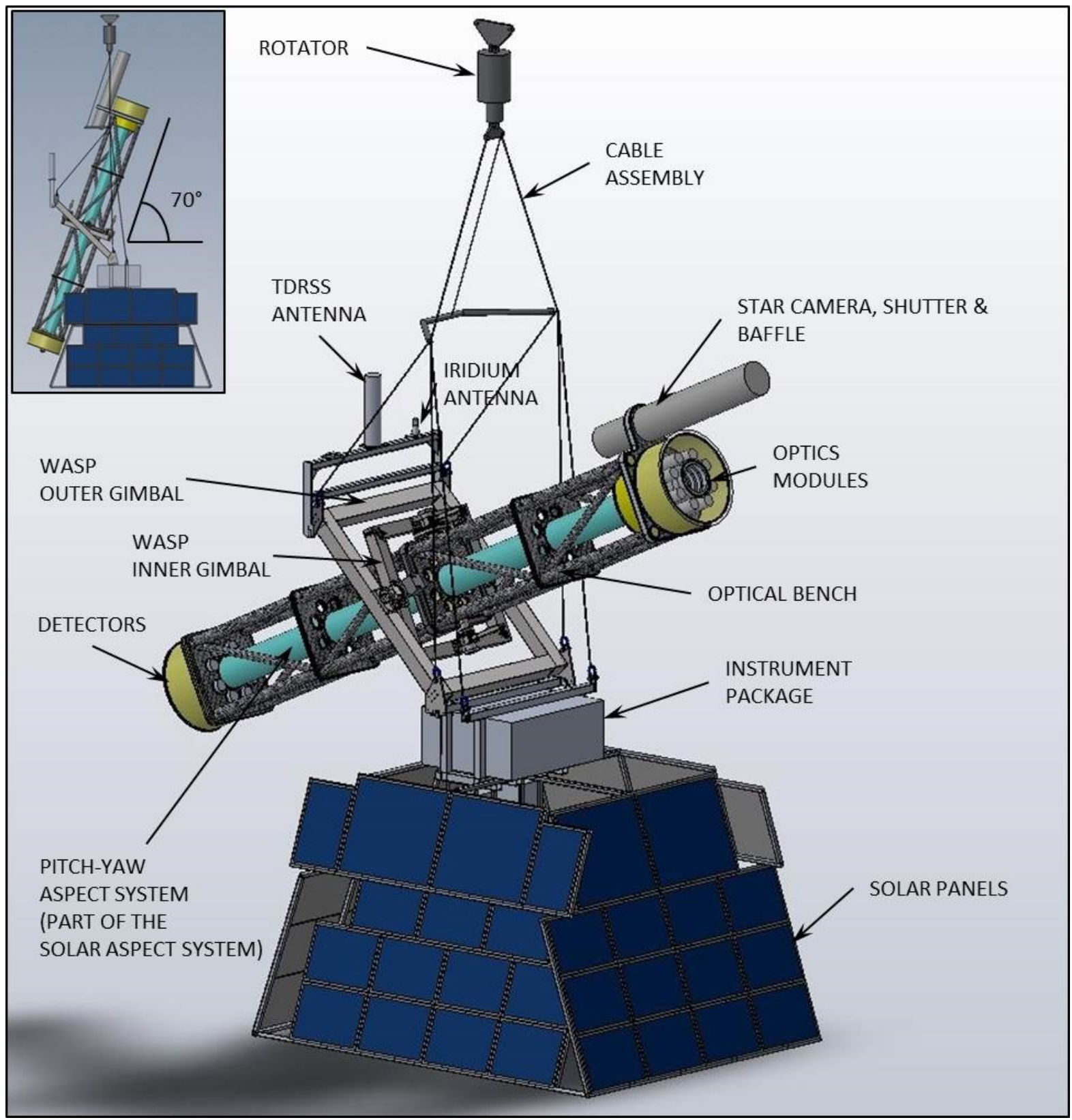

Figure 3. A diagram of the SuperHERO gondola and payload is shown with major components labeled. Additional space next to the Instrument Package is available for batteries and support electronics. The total weight of the science payload is $<2,500 \mathrm{lbs}$, appropriate for a LDB flight. The inset diagram shows the telescope pointed at a $70^{\circ}$ angle from the horizontal; the limit of our pointing capability due to interference from the balloon.

Prior to flight, extensive instrument calibration will take place. For the x-ray optics, this will involve pinhole scans at the MSFC Stray-Light Facility across the focal plane to determine effective area as a function of energy and angular resolution for a series of off-axis angles. Ray-trace models will be used to correct these data for infinite source distance. For the detectors, scans with mono-energetic x-ray beams will be used to generate response matrices for different energies and on-detector positions. Post flight, aspect information will be derived from the star-camera or solar aspect system images. For both, interpolation between aspect updates will be achieved by integrating gyro data from the inertial pointing system. A more extensive discussion of the pre-flight calibration process for HEROES, which will have a similar process to that of SuperHERO, can be found in reference [15].

The optics, detectors, their mounting and the system alignment will be discussed in detail the following sections. Table 2 gives a summary of the optics and detectors to be used on SuperHERO. 
Table 2. Summary of SuperHERO HXR Telescope.

\begin{tabular}{|c|c|}
\hline Key Component & Description \\
\hline Core Instrument & $\begin{array}{l}\text { HXR telescope consisting of high resolution optics (fabricated at MSFC) mated } \\
\text { to complimentary detectors (developed at RAL and GSFC). } \\
\qquad \quad \text { Energy Range: } \sim 20 \text { to } 76 \mathrm{keV} \text { (upper limit is due to Iridium K } \\
\text { edge) } \\
\text { - On-Axis Effective Area: } \sim 145 \mathrm{~cm}^{2} \text { at } 30 \mathrm{keV} \text { (total) } \\
\text { - } \quad \text { Field of view: } \sim 7 \text { arcmin FWHM at } 30 \mathrm{keV}\end{array}$ \\
\hline Optics & $\begin{array}{l}\text { Electroformed nickel-cobalt alloy mirror shells nested into multiple modules. } \\
\text { - Mirror Shells: } 152 \text { (19 shells arranged into } 8 \text { Modules) } \\
\text { - } \quad \text { Angular Resolution: } \sim 20 \text { arcsec HPD } \\
\text { - } \quad \text { Focal Length: 6-m } \\
\text { - Mirror Coating: Iridium (20 nm thick) } \\
\text { - Shell Thickness: } 250 \mu \mathrm{m}\end{array}$ \\
\hline Detectors & $\begin{array}{l}\text { These will be tiled into a } 2 \times 2 \text { array to cover the full field of view of each mirror } \\
\text { module. Active shielding will be used to reduce background. } \\
\text { - Detector Type: CdTe pixelated array } \\
\text { - Number of pixels: } 80 \times 80 \text { pixel arrays, } 3 \text {-side abuttable } \\
\text { - } \quad \text { Pixel Pitch: } 250 \mu \mathrm{m} \\
\text { - CdTe Thickness: } 1 \mathrm{~mm} \\
\text { - } \text { Energy resolution: } 1.3 \% \text { at } 60 \mathrm{keV} \text { (average pixel FWHM) } \\
\text { - Max processing rate: } 10 \mathrm{kHz} \text {, or }>5 \mathrm{M} \text { cts s over a } 80 \mathrm{x} 80 \text { detector } \\
\text { - } \text { ASIC can accommodate up to } 200 \mathrm{keV} \text { with thicker CdTe or CZT }\end{array}$ \\
\hline
\end{tabular}

\section{X-RAY OPTICS}

Optics Developments at MSFC

MSFC has been developing electroformed-nickel replicated (ENR) x-ray optics for more than decade. Nickel-cobalt alloy mirror shells are fabricated by electroforming onto a figured and super-polished aluminum mandrel plated with electroless nickel from which they are later released by differential thermal contraction [16], [17]. These are fullshell optics, and are inherently very stable, light weight and are suitable for operation in the HXR regime $(20-75 \mathrm{keV})$. These mirrors are in a Wolter Type I configuration, which requires 2 reflections to focus the incoming x-rays [18]. The first reflection is off of a parabolic shaped surface, and the second off of a hyperbolic surface. Figure 4 illustrates $\mathrm{x}-$ rays from an infinitely-distant source being focused by this type of $x$-ray optic. The incident $\mathrm{x}$-rays are nearly parallel to the surface of the mirror shells. These shallow graze angles make it difficult to build up large collecting areas (i.e., effective areas). To help with this problem, multiple mirror shells are nested inside one another (Figure 4, inset).

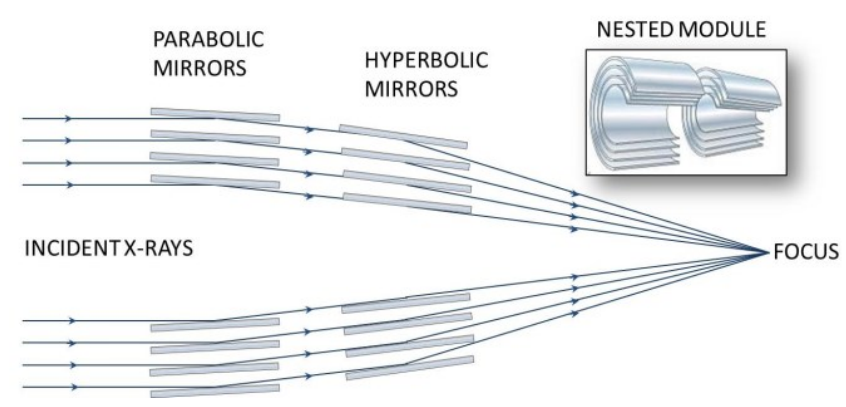

Figure 4. Schematic of a Wolter Type I configuration. To focus the incoming $x$-rays, they are reflected once off of a parabolic surface and then again off of a hyperbolic surface. To improve the collecting area of the telescope, multiple mirror shells are nested into modules (inset).

The type of mirror shell that SuperHERO will use was originally developed for the HERO payload. These mirror shells are conic approximations to Wolter Type I, and are single, full-shell structures (Figure 5). The first version of HERO consisted of two mirror modules, each containing three nested shells. This 3-m focal length telescope had an effective area of $2 \mathrm{~cm}^{2}$ at $40 \mathrm{keV}$ and $45 \operatorname{arcsec} \mathrm{HPD}$ for a module. Several phases of HERO followed later with improved optical configurations. 


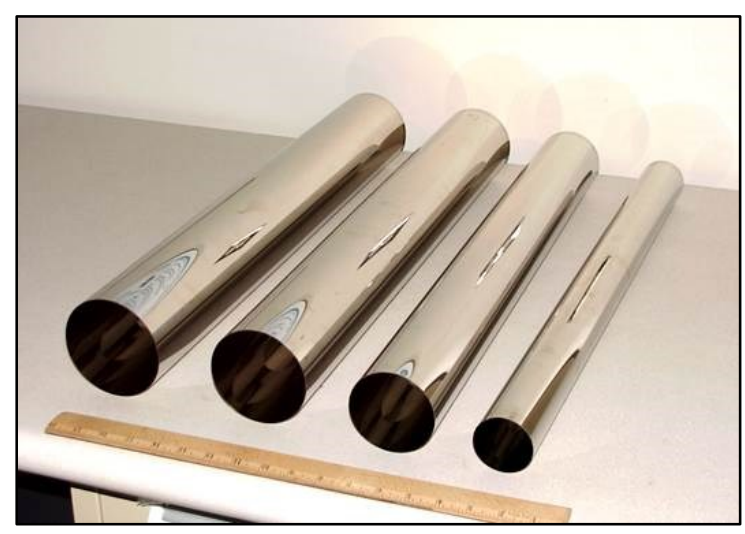

Figure 5. HERO individual mirror shells, which are conic approximations to a Wolter Type I configuration.

The most recent configuration is that of the HEROES payload. HEROES consists of 8 mirror modules housing a total of 109 grazing-incidence optics resulting in $85 \mathrm{~cm}^{2}$ effective area at $40 \mathrm{keV}$ and $40 \mathrm{~cm}^{2}$ at $60 \mathrm{keV}$, with an angular resolution of $\sim 25$ arcsecs HPD per module (Figure 6) [19].

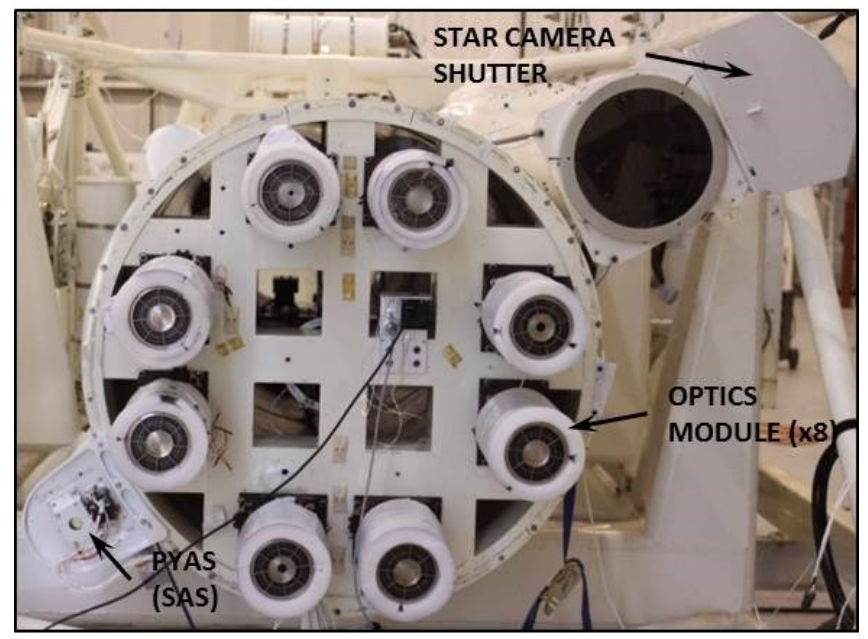

Figure 6. Image of the HEROES mirror modules mounted on the optical bench. HEROES has 8 mirror modules, each with 13 or 14 x-ray nested mirror shells. Also visible are the PYAS and star camera shutter to facilitate pointing during solar observations.

In addition to the HERO/HEROES optics, MSFC has been developing full-shell electroformed $\mathrm{x}$-ray mirrors for several collaborative efforts which include for Focusing Optics for $\mathrm{X}$-ray Solar Imaging (FOXSI), Micro-X and the Astronomical Roentgen Telescope X-ray Concentrator (ART-XC). FOXSI is a sounding rocket payload that flew in November 2012 and has observed several targets that include active regions, the quiet Sun, and a GOES-class B2.7 microflare. For the first time FOXSI captured focusing images of the Sun above $5 \mathrm{keV}$ [20]. The x-ray telescope that flew on this rocket payload is a 7 module system (each module housing 7 mirror shells) with a $2-\mathrm{m}$ focal length. Its nominal effective area is $100 \mathrm{~cm}^{2}$ at $10 \mathrm{keV}$ and $10 \mathrm{~cm}^{2}$ at
$15 \mathrm{keV}$ with a HPD value of 27 arcsecs on-axis (FWHM of 4.3 arcsecs on-axis) (Figure 7, lower-left). Optics are currently under fabrication for Micro-X, a MIT led rocket based payload which will consist of 0.5 meter diameter xray optics and a calorimeter detector. Schedule to fly in 2016; Micro-X aims to obtain images in the $0.2-3 \mathrm{keV}$ band of supernova remnants Puppis A and Cas A. MSFC has fabricated the ART-XC x-ray optics modules for launch on board the Spectrum Roentgen Gamma Mission (SRG). ART-XC consists of 7 modules with 28 nested shells in each, resulting in $450 \mathrm{~cm}^{2}$ effective area with the energy response up to $30 \mathrm{keV}$ (Figure 7, upper-left). Though required angular resolution of ART-XC is $<1$ arcmin, measured HPD of the mirror modules is better than 30 $\operatorname{arcsecs}[21]$.

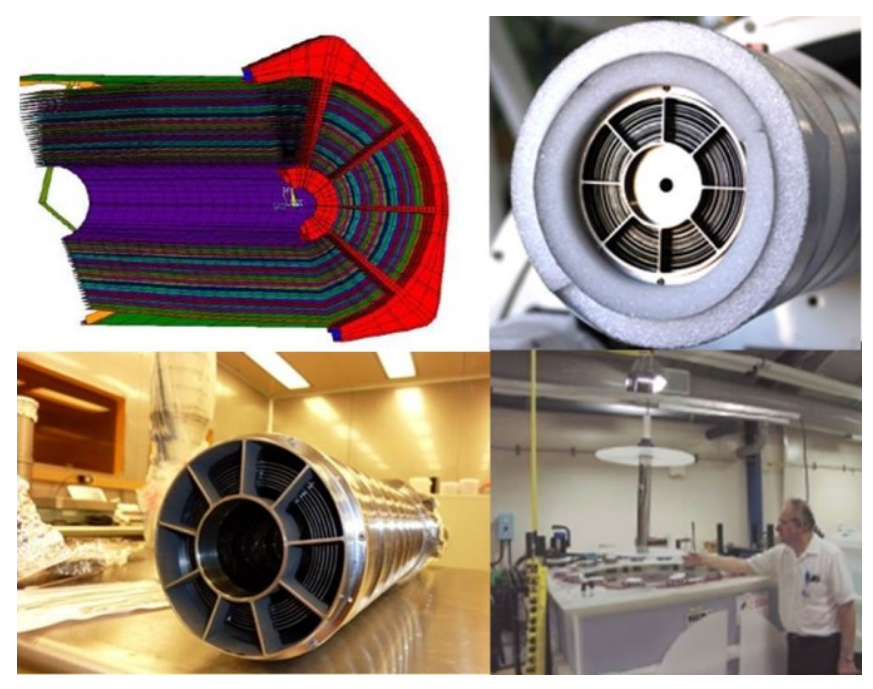

Figure 7. (top-left) Cross-section of an ART-XC module with 28 nested shells [22]. (upper-right) Image of a HEROES module with 14 mirror shells. (lower-left) Picture of an FOXSI module with 7 mirror shells mounted. (lower-right) Picture of the electroplating baths at MSFC for fabricating individual mirror shells.

SuperHERO will take advantage of these flight-proven optics developments, which have also lead to improved mounting and alignment techniques that are discussed in more detail in $\S 5$.

\section{SuperHERO Optics}

The SuperHERO mirror modules will be populated with the existing HEROES optics combined with additional mirror shells for increased effective area. The HEROES optical configuration has a total of 109 mirror shells assembled into 8 modules with the simulated effective area of $\sim 96 \mathrm{~cm}^{2}$ at $30 \mathrm{keV}$ and $\sim 88 \mathrm{~cm}^{2}$ at $40 \mathrm{keV}$. The mirror shell mounting structure obscures roughly $4.7 \%$ of the effective area, which translates to a usuable effective area of $\sim 84 \mathrm{~cm}^{2}$ at $40 \mathrm{keV}$ (consistent with measured values). A trade-study was performed to compare the effective area, mass, and cost associated with adding mirror shells to the existing HEROES modules to just adding 2 additional HEROES 
modules. We determined that adding 43 mirror shells to the existing HEROES compliment (i.e., giving 19 mirror shells in each of the 8 modules) was the optimal solution. This will increase the effective area by $\sim 50 \%$ at $30 \mathrm{kev}$ and by $\sim 40 \%$ at $45 \mathrm{keV}$ (Figure 8). The trade-off to adding these mirror shells is that the total mass of the optics (and hence the total payload) will be increased by $14 \mathrm{~kg}$, which should not significantly impact the duration of the LDB flight (of which payload mass is one factor.)

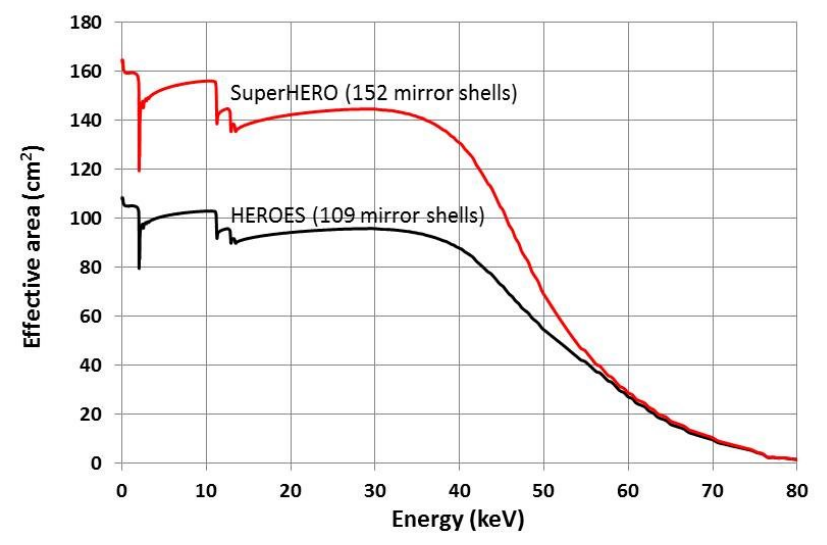

Figure 8. Simulated total effective area for the SuperHERO (upper curve) and the HEROES configuration (lower curve).

A second consideration when determining the optimum mirror shell configuration is the field of view (FOV). The FOV for SuperHERO is mainly due to the response of the mirror shells to an off-axis source. The configuration chosen for the SuperHERO balloon payload has a calculated FOV of 7 arcmins FWHM at $30 \mathrm{keV}$ (Figure 9).

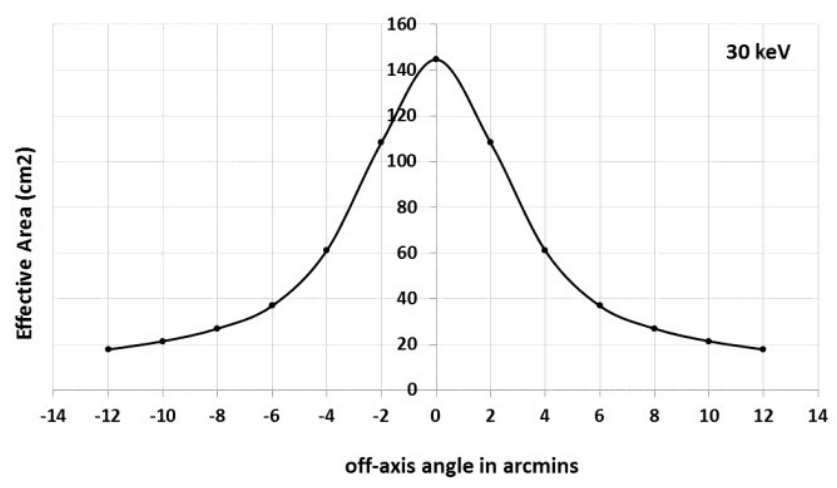

Figure 9. FOV response at $30 \mathrm{keV}$ for the SuperHERO mirror configuration. This plot includes contributions from all 152 mirror shells and shows how the effective area falls off as a function of off-axis angle. The FOV at $30 \mathrm{keV}$ is 7 arcmin FWHM.

Prior to assembly on the SuperHERO gondola, the mirror shells will be remounted and realigned using new techniques developed at MSFC, for improved module angular resolution of $\sim 20$ arcsecs HPD (discussed in more detail in $\S 5$ ). Angular resolution directly relates to the Point Spread Function (PSF), which is a measure of the total flux fraction for a point-source. Figure 10 compares the integrated Point Spread Function (PSF) of NuSTAR, whose angular resolution is 58 arcsec HPD [23] to SuperHERO's $\sim 20$ arcsec HPD expected performance.

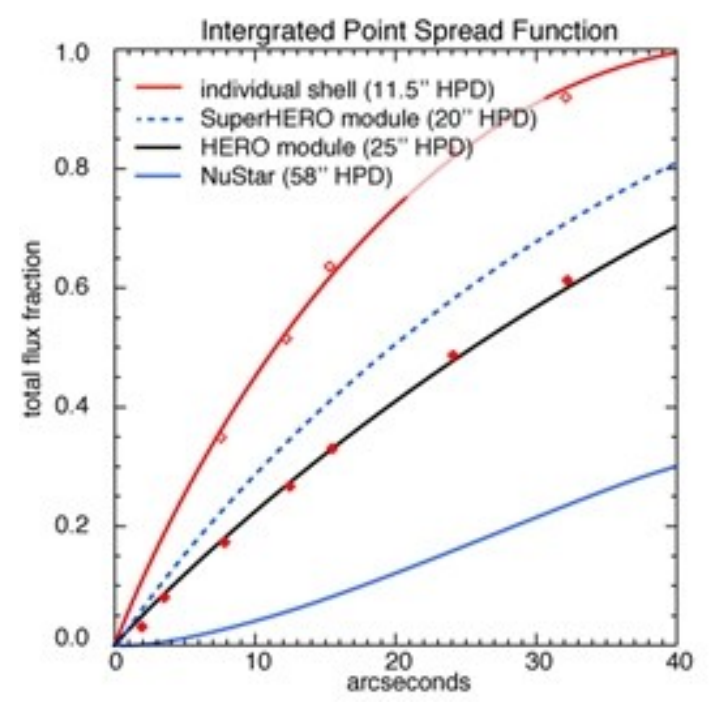

Figure 10. The integrated PSF of the same mirror module as compared to a SuperHERO 20 arcsec HPD is shown compared to that of the NuSTAR telescope.

Implementation of this new alignment technique, combined with the intrinsic properties of the ENR optics developed under the HERO program will provide the highest resolution currently available in HXR optics.

\section{COMPLIMENTARY DETECTORS}

\section{CdTe Multi-Pixel Detectors}

High-angular-resolution HXR optics require detectors with a large number of fine and independent pixels in order to adequately sample the telescope PSF over the entire field of view. Excessively over-sampling the PSF will increase readout noise and require more processing with no appreciable increase in image quality. An appropriate level of over-sampling is to have 2-3 pixels within the HPD. The SuperHERO optics are expected to have an HPD of 20 arcsec over a 6-m focal length, which converts to $600 \mu \mathrm{m}$ at the focal plane. A detector with a pixel size of $250 \mu \mathrm{m}$ will oversample the optics by 2.4 times, which is within the desired range. Of course, the detectors must also have high efficiency in the HXR region, good energy resolution, low background, low power requirements, and low sensitivity to radiation damage [24]. The ability to handle high counting rates is also necessary for solar observations.

Over the past decade or so, Rutherford Appleton Laboratory (RAL) in the UK has been developing detectors that are well suited to appropriately oversample the SuperHERO optics. Dubbed HEXITEC, for High Energy X-Ray Imaging Technology, this Application Specific Integrated Circuit (ASIC), can be bonded to 1- or 2- mm-thick cadmium 
telluride (CdTe) or up to 5- mm-thick cadmium-zinctelluride (CZT), to create a fine pixel (250 $\mu \mathrm{m}$ pitch) HXR detector [25], [26]. These detectors are roughly $2 \mathrm{~cm} \times 2 \mathrm{~cm}$ in size and have $80 \times 80$ pixels. To fill the full field of view of the SuperHERO mirrors, 4 detectors will be arranged into a $2 \times 2$ array (Figure 11). Each SuperHERO mirror module will have its own 2 × 2 detector array. These detectors have been tested extensively in a laboratory environment [26], [27], [28] and larger arrays (5x5) have been assembled, with good results [29].

The SuperHERO team is in the process of readying these detectors for flight (balloon and sounding rocket). The frontend electronics instrument boards, including Field Programmable Gate Arrays combined with analog amplifiers and high-speed, low-power ADCs, power regulation, temperature monitors, data storage, and a communication interface for use with HEXITEC-based CdTe detectors are being developed and tested. In parallel, a thermal stabilization system is being developed to maintain the detectors at the optimum $-20^{\circ} \mathrm{C}$ operating temperature. The detectors will also be calibrated using X-rays, and tested in a vibration and environmental facility at MSFC.

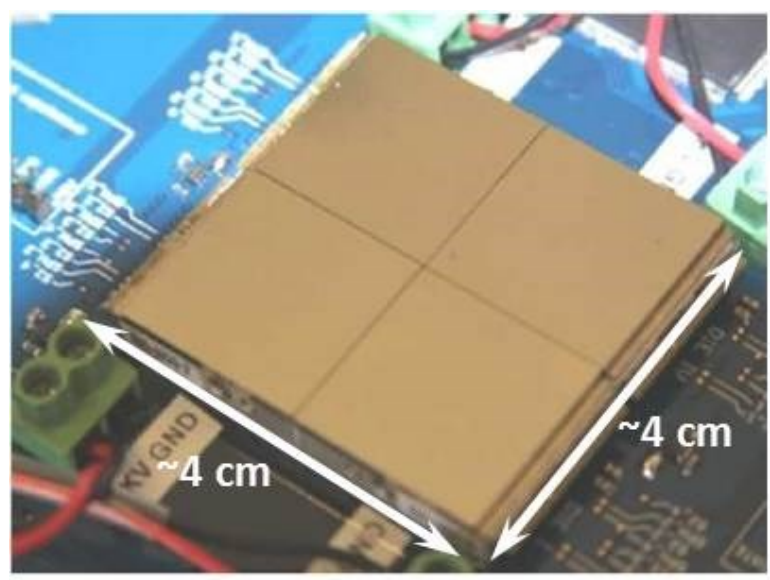

Figure 11. A $2 \times 2$ arrangement of the HEXITEC detectors showing how closely they can abut with each other is shown. Each detector has $80 \times 80$ pixels with 250 $\mu \mathrm{m}$ pitch [28].

\section{Detector Mounting}

The $2 \times 2$ detector arrays will be hard-mounted (i.e., no adjustability) at the focal plane, located $6 \mathrm{~m}$ from the optics. These will be surrounded by a graded-Z passive shield and a plastic-scintillator active shield. The passive shield, primarily composed of lead, attenuates photons from all directions outside the FOV of the grazing-incidence optics. In addition to non-local photon background, cosmic rays interacting with the instrument, especially in the passive shield, produce secondary photons locally. The active shield surrounding the passive shield detects the passage of cosmic rays and operates in anti-coincidence with the $\mathrm{CdTe}$ detectors to veto out such locally produced background. The active shield is composed of two coupled pieces of plastic scintillator in light-tight housing, read out by a single 3"-diameter PMT.

Each detector assembly and its shield will reside in an aluminum pressure vessel filled with dry nitrogen to mitigate arcing, condensation, and aid heat dissipation. A thin aluminum window will allow X-rays to reach the detectors and prevent visible sunlight from reaching the detector. The instrument board will also be enclosed within a pressure vessel.

\section{SYSTEM ALIGNMENT}

Alignment of the mirror modules to the detectors will be fixed by their mechanical mounting to the optical bench. The mirror modules and detectors will be mounted to aluminum honeycomb core plates located at the front and focal plane of the optical bench respectively.

Steel inserts in the sandwich plate will provide the interface to the mirror modules. These will be ground flat and spherical washers will be used to attach to the three mounting lugs of the mirror spider. The use of this type of three-point mount avoids distorting the spider when it is bolted down and this in turn prevents any distortions from being imparted on the mirror shells themselves. The use of a sophisticated alignment system ensures that the system optical axis is normal to the plane of the spider. Thus only small alignment adjustments are necessary when mounting and these can be accomplished through the use of shims.

\section{Science Requirements}

The principal requirement for alignment is that the optics be close to the center of their response curve when pointed at the target. This means in practice that the optics need to be coaligned to about $+/-0.5$ arcmin with respect to the aspect system. This level of accuracy can be achieved with an alignment fixture placed some distance from the payload and a portable x-ray system to illuminate the x-ray mirror modules. Such a system also enables the optics to detector alignment to be determined, although this can also be verified during flight using bright cosmic sources. Typical machine tolerances should be more than adequate to achieve the desired alignment with little adjustment, although the possibility of changes in alignment due to changing conditions in flight are always present.

\section{Balloon Platform Considerations}

The two main considerations required for maintaining mirror to detector alignment is structural integrity of the optical bench and thermal management throughout the flight. The SuperHERO optical bench is designed to be mechanically strong and have near zero CTE. Despite this, there will be some sagging of the optical bench due to gravity and possibly some bowing of the optical bench (and components) due to thermal variations over the course of the 2 to 4 week long flight. 
To mitigate source blurring due to deflections in the optical bench during flight, SuperHERO will incorporate the flight proven, Bench Alignment Monitoring System (BAMS). On HEROES, BAMS used 2 cameras located at the elevation flange, at the center of the optical bench, to continuously image rings of LEDs located on both the $\mathrm{x}$-ray mirror mounting plate and detector mounting plate. This system on HEROES was able to measure relative angular deflections to better than 10 arcsecs. However, due to the BAMS mounting scheme on HEROES, there is a large uncertainty in the measurement. For use on SuperHERO, the optimal mounting positions for the CCDs and LED rings are still being assessed.

Deflections due to thermal variations will be mitigated by wrapping the mirror modules with heater tape that can either be actively controlled via an onboard thermostat, or through manual control on the ground. Each mirror module will be insulated with open-cell foam. Multi-Layered Insulation (MLI) may be used as additional passive mitigation against radiative heat transfer. Thermal management of the detector plane requires a more detailed design of the detector electronics and anticoindence shielding, mounting and geometry than what has currently been carried out. This work will be done at a future time.

\section{X-Ray Mirror Module Assembly}

The HERO/HEROES individual mirror shells have typical angular resolutions of $\sim 15$ arcsecs HPD (and as good as 11.5 arcsecs), but the HERO/HEROES modules had $\sim 25$ arcsec resolution due to mounting errors. For SuperHERO, the plan is to draw on experience gained with the ART, FOXSI [30] and FOXSI-2 programs and change both the design philosophy of the mirror module and the technique used to align and mount the individual shells within it. In this way, we will improve the overall module quality to $\sim 20$ arcsec HPD. This will allow us to leverage the improved pointing capability of WASP, and the finer spatial resolution of the CdTe detectors $(250 \mu \mathrm{m})$ which will oversample the mirror response, to give an overall system resolution of $\sim 22$ arcsecs HPD.

For the ART-XC program, a single spider was used to support the mirror shells and to attach the mirror module to its support plate. This approach has several advantages over the traditional approach where the mirror shell is held at both ends. It avoids over-constraining the shell figure which can happen when both ends are trying to define and control the circularity of the shell. It also obviates the need to accurately select the expansion coefficient of the housing body material to avoid distorting the captive shells when the module temperature changes and somewhat relaxes the temperature control requirements. It also permits the use of much lighter materials for the housing body as it is no longer a structural element responsible for transferring loads.

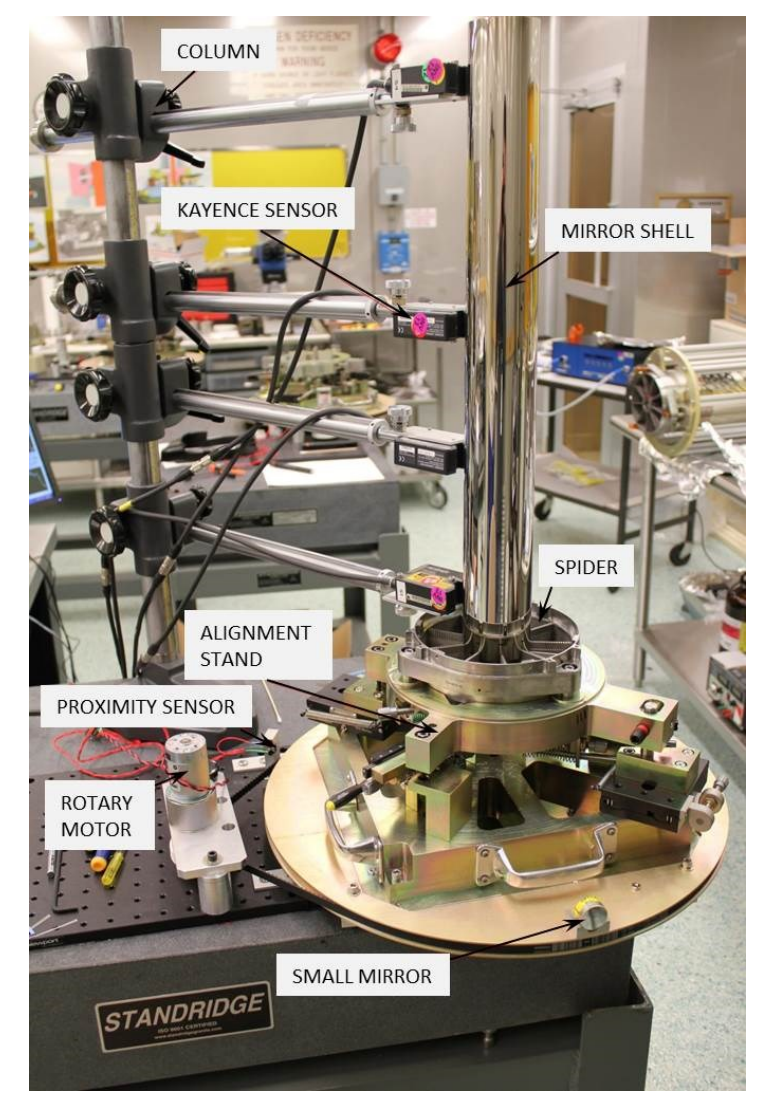

Figure 12. Image of a single $x$-ray mirror shell being aligned and mounted into a spider. The spider has grooves into which single shells are mounted. Once the shells are aligned, a low-shrinkage epoxy is injected into the groove to fix the shell in place.

The shell alignment and mounting approach, used for both the FOXSI [30] and ART [31] programs, makes use of adjustable 3-point mounts which are used to support each successive shell during integration. During this operation the shell is monitored by displacement sensors at multiple positions along its length, the system is rotated through 360 degrees in front of the sensors, and the mounts are adjusted to get the shell circular and concentric with the spider assembly. At this point the shells are floating in the spider grooves, but when the software indicates that the mounting errors are within an acceptable range, low-shrinkage epoxy is injected into the groove (Figure 12). For the FOXSI Programs, which had a tighter resolution requirement, a UV-cure epoxy was used underneath the shell first to pin its position and minimize later epoxy-shrinkage effects, whereas for ART a standard room-temperature epoxy was used to fill the groove in one go. Using this technique FOXSI-2 [32] achieved a HPD of $\sim 20$ arcsecs and 6-7 arcsecs FWHM.

\section{THE FUTURE}

\section{Optics Developments}

MSFC is continuously working to improve the quality of their x-ray mirrors (fabrication, assembly, metrology and 
testing). In order to meet the future astronomical need for high quality optics MSFC is currently pursuing various aspects of technology development including: new mirror fabrication approaches; techniques for figure correction for significantly improving mirror resolution; efficient and accurate mirror metrology; and multilayer coatings to extend the energy bandwidth of grazing-incidence optics.

In addition to improving its standard electroforming process, MSFC is developing the capability to directly figure and polish full-shell glass and metal optics. This approach, similar to that used to fabricate the mirrors for the Chandra observatory, makes use of new developments in computer-controlled polishing machines, in this case a Zeeko seven axis IRP 600, and novel approaches for mounting and handling very-thin shell optics. The goal is to develop light-weight full-shell optics with $\sim 5$ arcsec HPD angular resolution, which can then be further improved with another in-house development -- differential deposition. This technique involves a preferential coating mechanism that does selective deposition on the surface of the mirror to improve its figure. Preliminary studies at MSFC have shown very promising results and the infrastructure has recently been put in place to extend this study beyond the proof of concept stage [33].

The quality of mirrors is only as good as the supporting metrology. Roughly half of the time in mirror development is spent in metrology and so increasing the speed of such measurements can have significant benefits. The current MSFC 'workhorse' is the slope-measuring long trace profiler [LTP]. This equipment is capable of measuring the surface profile with a slope resolution of $<1 \mu \mathrm{rad}$, enough to satisfy current needs but its single-beam approach makes large-area measurements very time consuming. In order to improve the metrology efficiency, a new multi-beam LTP is currently under development which works on the same principle of LTP but instead of a single laser beam, uses 10 beams to scan the test surface simultaneously, which will decrease the time required for a single scan by almost an order of magnitude. [34].

Finally, the highest energies achievable in current MSFC optics is set to $\sim 75 \mathrm{keV}$ by the iridium mirror-shell coatings that are typically used. Activities are underway to develop multilayers coatings that will give useful reflectivities to much higher energies $-150-200 \mathrm{keV}$. Coating chambers are currently being constructed to investigate promising material combinations to produce these high-energy coatings.

\section{Detector Development}

The current HEXITEC ASIC is designed to accommodate $250 \mu \mathrm{m}$ pixels, which is sufficient to adequately oversample the existing HEROES/SuperHERO optics at a 6-m focal length. As MSFC develops higher resolution optics with a goal of 10 arcsec HPD or better, detectors with a finer pixel pitch would be of benefit. RAL is currently capable of fabricating indium bond pads of $20 \mu \mathrm{m}$ or smaller. Using a $180 \mathrm{~nm}$ CMOS Image Sensor process (from TowerJazz Semiconductor), it should be possible to fabricate detectors with a pixel pitch of $\sim 150 \mu \mathrm{m}$. Though this would require modifications to the existing HEXITEC ASIC and potentially significant cost, we have not ruled out the future use of such detectors. The alternative to a finer pixel pitch is a longer focal length. Mirror shells with an angular resolution of 10 arcsec, for example, which MSFC is ontrack to develop, and a focal length of 10-m (compared to the current 6-m focal length of SuperHERO), the $250 \mu \mathrm{m}$ pixel pitch offered by the HEXITEC detectors would oversample the optics by a factor of $\sim 2$. As expected, a 20$\mathrm{m}$ focal length would oversample the optics by a factor of $\sim 4$.

Given the possibility of higher angular resolution HXR mirrors from MSFC and the fine-pitch pixel detectors from RAL, the SuperHERO team has started to consider possible configurations for a future space-based mission.

\section{SuperHERO Explorer Mission}

The future of HXR astronomy lies in the ability to fly a high resolution spectroscopic and imaging focusing telescope on a satellite platform. To this end, we have started looking into possible mission configurations for which the SuperHERO balloon payload can evolve into. As we refine potential mission science goals, the telescope configuration will be refined as well. As such, this is a very preliminary configuration.

This configuration would consist of 3 modules, each with 45 nested mirror shells, with an angular resolution as good as 10 to 20 arcsecs. The telescope would have a $20-\mathrm{m}$ focal length and would utilize the HEXITEC detectors, with 250 $\mu \mathrm{m}$ pitch pixels. The mirror shells would range in diameter from $\sim 16 \mathrm{~cm}$ to $\sim 26.9 \mathrm{~cm}$ (Figure 13). These shells would be closely packed and would be coated with Iridium. Multilayers will also be considered, depending on the maturity of the process at the time of fabrication.

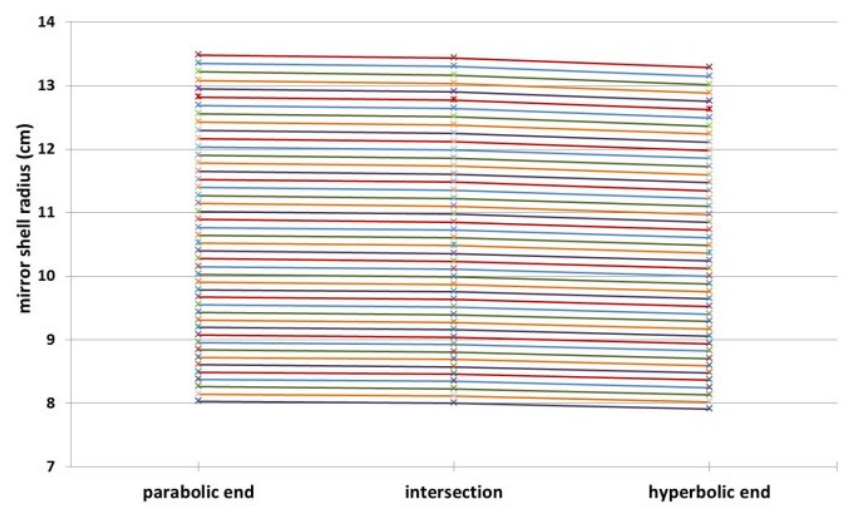

Figure 13. The SuperHERO satellite configuration would consist of 3 modules, each with 45 nested mirror shells. The radius of each shell is plotted, illustrating the closely-packed nesting structure for a single module. 
This HXR telescope would have a total on-axis effective area of $336 \mathrm{~cm}^{2}$ at $30 \mathrm{keV}$, which is comparable to that of NuSTAR [3], and FOV of $\sim 7$ arcmins (FWHM) at $30 \mathrm{keV}$. Figures 14 and 15 show the simulated effective area and field of view as a function of energy, respectively, for the proposed configuration. Figure 14 also illustrates the mirror response as a function of off-axis angle of the source relative to the optic.

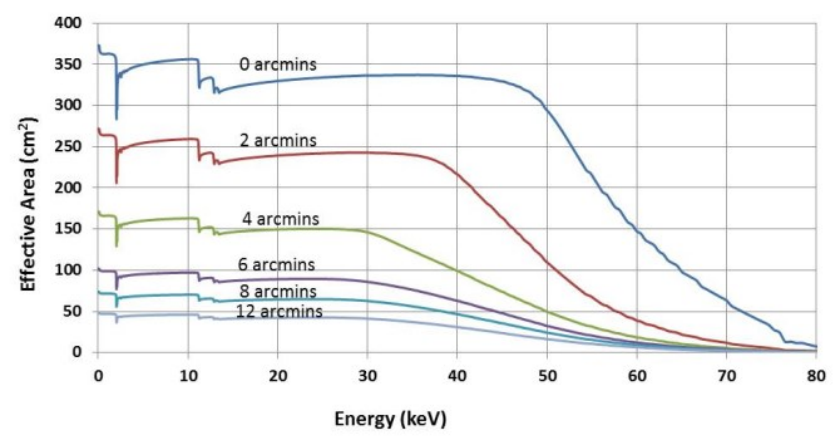

Figure 14. Plot of the simulated total effective area for the SuperHERO space-based optics configuration. The top curve is the on-axis response ( 0 arcmins off-axis), and the subsequent curves are the responses for a source that is $2,4,6,8$ and 12 arcmins off-axis.

As can be seen from this Figure, the effective area falls off rapidly as a function of off-axis angle. This is mainly due to vignetting caused by the closely packed mirror shells. Similarly, the FOV (Figure 15) also falls off rapidly as a function of energy.

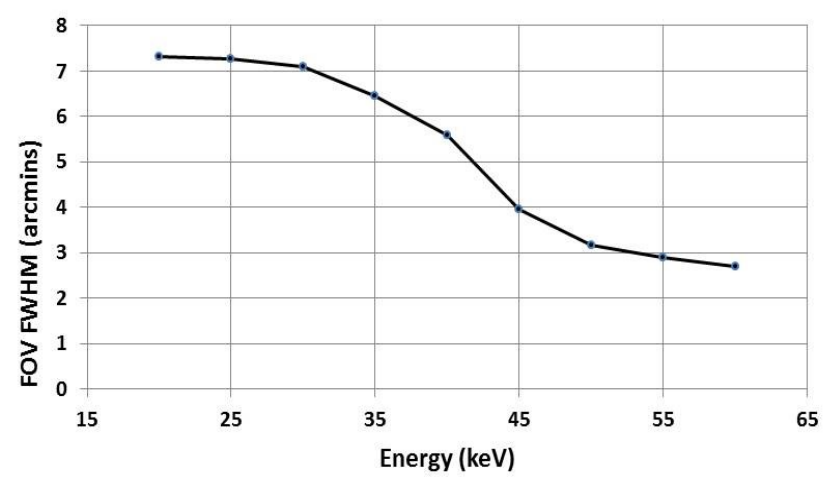

Figure 15. Plot of the FOV as a function of energy for a SuperHERO satellite module.

The estimated mass of the $\mathrm{x}$-ray mirrors is $147 \mathrm{~kg}$ for all 3 modules, which would most likely require a NASA MidExplorer (MidEx) class mission to accommodate. A MidEx, or Medium-Class Explorer, is capable of supporting payloads with a total mass of around $661 \mathrm{~kg}(1,457 \mathrm{lbs})$ [35]. The estimate for the mirror mass assumes a simplified mirror shell thickness of $300 \mu \mathrm{m}$ and does not include mounting structures.

The configuration for a SuperHERO explorer payload is driven by the science goals, which currently include astronomical targets such as the Galactic Center, pulsarpowered synchrotron nebulae and diffusive shock accelerated sites in supernova remnants science-driven, and the Sun. As these goals become more refined, we expect the configuration to change appropriately. This first exercise proves the feasibility of using the MSFC optics to build up a comparable effective area to that of NuSTAR, and existing HXR satellite mission, and to estimate the required platform (MidEx as opposed to a NASA Small Explorer Mission) to manage the mass constraints of this payload.

\section{SUMMARY}

The SuperHERO balloon payload is a HXR telescope, currently in its proposal phase that is intended to fly on a LDB flight from Antarctica with the intent of observing astronomical targets and the Sun during the same flight. The telescope has a 6-m focal length and consists of 8 individual mirror modules, each of which is coupled to a $2 \times 2$ array of fine pixel CdTe detectors from RAL. These novel detectors facilitate the use of the high angular resolution optics developed at MSFC; resulting in the highest angular resolution HXR telescope in the present day. These detectors have comparable energy resolution to other, similar devices with the added advantages of small pixel pitch $(250 \mu \mathrm{m})$, a high maximum processing rate of $10 \mathrm{kHz}$ and a modular configuration necessary for covering large focal planes.

The HERO, HEROES and SuperHERO balloon payloads are stepping stones to an eventual NASA Explorer-class, space-based, mission. One of the primary advantages of the balloon platform is that it offers a challenging, space-like environment on which one can prove a concept and increase the Technology Readiness level (TRL) of an instrument to a point at which it can be flown in space on a satellite. Several balloon payloads have evolved in this way. Some of these include: High Energy Focusing Telescope (HEFT) to NuSTAR [36], the Cosmic Ray Energetics and Mass (CREAM) to International Space Station-CREAM [37], Burst and Transient Source Experiment (BATSE) to BATSE on the Gamma Ray Observatory [38].

The SuperHERO Explorer payload current configuration consists of 135 x-ray mirror shells configured into 3 modules (45 shells per module). The focal plane detectors would be the same as those used for the SuperHERO balloon flight. The focal length would be extended to $20-\mathrm{m}$ to take full advantage of the high resolution optics. As the configuration is science-driven, we expect modifications as the science goals continue to be refined.

\section{ACKNOWLEDGEMENTS}

The authors would like to thank NASA MSFC and GSFC internal Center support for the design of the SuperHERO gondola and detector developments, respectively. We would also like to thank the NASA competed Astrophysics 
Research and Analysis Program for supporting the maturation of the detectors for flight (NNH13ZDA001NAPRA, Program Manager: Louis J. Kaluzienski).

\section{REFERENCES}

[1] S. O'Dell, R. Brissenden, W. Davis, R. Elsner, M. Elvis, M. Freeman, T. Gaetz, P. Gorenstein, M. Gubarev, D. Jerius, M. Juda, J. Kolodziejczak, S. Murray, R. Petre, W. Podgorski, B. Ramsey, P. Reid, T. Saha, D. Schwartz, S. Trolier-McKinstry, M. Weisskopf, R. Wilke, S. Wolk and W. Zhang, "Highresolution x-ray telescopes," in Proc. SPIE 7803, $78030 H, 2010$.

[2] M. Weisskopf, H. Tananbaum, L. Van Speybroeck and S. O'Dell, "Chandra X-ray Observatory (CXO): overview," in Proc. SPIE 4012, Munich, Germany, 2000.

[3] F. Harrison, W. Craig, F. Christensen and NuSTAR Team, "The Nuclear Spectroscopic Telescope Array (NuSTAR) High Energy X-Ray Mission," ApJ, vol. 770, p. 103, 2013.

[4] C. a. JPL, "NuSTAR: Bringing the High Energy Universe into Focus," [Online]. Available: http://www.nustar.caltech.edu/. [Accessed 3 October 2014].

[5] B. D. Ramsey, C. D. Alexander, J. A. Apple, C. M. Benson, K. L. Dietz, R. F. Elsner, D. E. Engelhaupt, K. K. Ghosh, J. J. Kolodziejczak, S. L. O'Dell, C. O. Speegle, D. A. Swartz and M. C. Weisskopf, "First Images from HERO, a Hard X-Ray Focusing Telescope," ApJ, vol. 568, pp. 432-435, 2002.

[6] D. A. Swartz, B. D. Ramsey, A. F. Tennant, K. L. Dietz, J. A. Apple, J. A. Gaskin and M. C. Weisskopf, "Resolving the Crab Nebula with Direct Hard X-ray Imaging," in $A A S$ HEAD 10th Divisional Meeting, Abstract \#31.06, San Grancisco, CA, 2008.

[7] S. Christe, A. Shih, M. Rodriguez, K. Gregory, A. Cramer, M. Edgerton, J. Gaskin, B. O'Connor and A. Sobey, "A Solar Aspect System for the HEROES Mission," in IEEE Aerospace Conference, Big Sky, MT, 2014.

[8] C. Wilson-Hodge, J. Gaskin, S. Christe, A. Shih, D. Swartz, A. Tennant and B. Ramsey, "Astrophysical Observations with the HEROES Balloon-borne Payload," in AAS 223rd Meeting, Washington, DC, Jan 05, 2014.

[9] J. Gaskin, S. Christe, R. Elsner, K. Kilaru, B. Ramsey, P. Seller, A. Shih, D. Stuchlik, D. Swartz, A. Tennant, B. Weddendorf, M. Wilson and C. Wilson-Hodge, "SuperHERO: the next generation hard $\mathrm{x}$-ray HEROES telescope," in Proc. SPIE, 9144, 91443Z, Montreal, Quebec, Canada, 2014.

[10] "Wallops Arc Second Pointer," 2014. [Online]. Available:

http://sites.wff.nasa.gov/balloons/technology_wasp_d etails.html. [Accessed 2014].

[11] "Columbia Scientific Balloon Facility," [Online]. Available:

http://towerfts.csbf.nasa.gov/Maps/ConvGps650N.ht m. [Accessed 2014].

[12] J. Gaskin, C. Wilson-Hodge, B. Ramsey, J. Apple, K. Dietz, A. Tennant, D. Swartz, A. Shih and S. Christe, "High Energy Replicated Optics to Explore the Sun Balloon-Borne Telescope: Astrophysical Pointing," in IEEE Aerospace Conference Proc., Big Sky, MT, 2013.

[13] J. Rhodes, B. Dobke, J. Booth, R. Massey, K. Liewer, R. Smith, A. Amara, J. Aldrich, J. Berge, N. Bezawada, P. Brugarolas, P. Clark, C. Dubbledam, R. Ellis, C. Frenk, A. Gallie, A. Heavens, D. Henry, E. Jullo, T. Kitching, J. Lanzi, S. Lilly, D. Lunney, S. Miyazaki, D. Morris, C. Paine, J. Peacock, S. Pellegrino, R. Pittock, P. Pool, A. Refregier, M. Seiffert, R. Sharples, A. Smith, D. Stuchlik, A. Taylor, H. Teplitz, R. Vanderveld and J. Wu, "Spacequality data from balloon-borne telescopes: the High Altitude Lensing Observatory (HALO)," Astroparticle Physics, vol. 38, pp. 31-40, 2012.

[14] Q. Guo, M. Beilicke, A. Garson, F. Kislat, D. Fleming and H. Krawczynski, "Optimization of the design of the hard X-ray polarimeter X-Calibur," Astroparticle Physics, vol. 41, pp. 63-72, 2013.

[15] C. Wilson-Hodge, J. Gaskin, S. Christe, A. Shih, A. Tennant, D. Swartz, K. Kilaru, R. Elsner, J. Kolodziejczak and B. Ramsey, "Calibration of the High Energy Replicated Optics to Explore the Sun (HEROES)," Journal of Astronomical Instrumentation, 2014 (In Press).

[16] B. Ramsey, R. Elsner, D. Engelhaupt, M. Gubarev, J. Kolodziejczak, S. O'Dell, C. Speegle and M. Weisskopf, "The Development of Hard X-Ray Optics at MSFC," in Proc. SPIE 5168, 2004.

[17] B. Ramsey, R. Elsner, D. Engelhaupt, S. O'Dell, C. Speegle and M. Weisskopf, "Development of Hard XRay Optics at MSFC," in Proc. SPIE 4851, 2003.

[18] M. K. Joy and B. D. Ramsey, "X-Ray Optics," in Handbook of Optics, Third Edition Volume $V$ : Atmospheric Optics, Modulators, Fiber Optics, X-Ray Optics and Neutron Optics, New York, McGraw-Hill, 2009, pp. 47.1-47.12.

[19] J. Gaskin, J. Apple, K. Stevenson Chavis, M. Holt, H. Koehler, T. Lis, B. O'Connor, M. Rodriguez-Otero, J. Pryor, B. Ramsey, M. Rinehart-Dawson, L. Smith, A. Sobey, C. Wilson-Hodge, S. Christe, A. Shih, A. Cramer, M. Edgerton, M. Rodriguez, A. Shih, D. Gregory, J. Jasper and S. Bohon, "High Energy Replicated Optics to Explore the Sun: Hard X-Ray Balloon-Borne Telescope," in IEEE Aerospace Conference Proc., Big Sky, MT, 2013.

[20] S. Krucker, S. Christe, L. Glesener, S.-n. Ishikawa, B. Ramsey, T. Takahashi, S. Watanabe, S. Saito, M. 
Gubarev, K. Kilaru, H. Tajima, T. Tanaka, P. Turin, S. McBride, D. Glaser, J. Fermin, S. White and R. Lin, "First Images Form the Focusing Optics X-ray Solar Imager," ApJ Letters, vol. 793, no. 2, p. L32, 2014.

[21] M. Gubarev, B. Ramsey, R. Elsner, S. O'Dell, J. Kolodziejczak, J. McCracken, V. Zavlin, D. Swartz, K. Kilaru, C. Atkins, M. Pavlinsky, A. Tkachenko and I. Lapshov, "ART-XC/SRG: Status of the X-Ray Optics Development," in Proc. SPIE 9144, 9144V, 2014.

[22] M. Pavlinsky, V. Akimov, V. Levin, I. Lapshov, A. Tkachenko, N. Semena, V. Arefiev, A. Glushenko, A. Yaskovich, R. Burenin, S. Sazanov, M. Revnivtsev, M. Buntov, S. Grebenev, A. Lutovinov, M. Kudelin, S. Grigorovich, D. Litvin, V. Lazarchuk, I. Roiz, M. Garin, M. Gubarev, B. Ramsey, K. Kilaru, S. O'Dell and R. Elsner, "The ART-XC Instrument On-Board the SRG Mission," in Proc. SPIE 8147, 814706, 2011.

[23] F. Harrison, in Proc. SPIE 7732, 27, 2010.

[24] B. Ramsey, IEEE Nuc. Sci. Symp. Conf. Rec., vol. 4, pp. R10-1, 2001.

[25] L. Jones, P. Seller, M. Wilson and A. Hardie, "HEXITEC ASIC - A Pixellated Readout Chip for CZT Detectors," NIM A, vol. 604, no. 1-2, pp. 34-37, 2009.

[26] P. Seller, S. Bell, R. J. Cernik, C. Christodoulou, C. Egan, J. Gaskin, S. Jacques, S. Pani, B. Ramsey, C. Reid, P. Sellin, J. Scuffham, R. Speller, M. Wilson and M. Veale, "Pixellated $\mathrm{Cd}(\mathrm{Zn}) \mathrm{Te}$ high-energy Xray instrument," JINST, vol. 6, p. C12009, 2011.

[27] M. Veale, S. Bell, D. Duarte, A. Schneider, P. Seller, M. Wilson and K. Iniewski, "Measurements of charge sharing in small pixel CdTe Detectors," NIM $A$, vol. 767, pp. 218-226, 2014.

[28] M. Wilson, S. Bell, R. Cernik, C. Christodoulou, C. Egan, D. O'Flynn, S. Jacques, S. Pani, J. Scuffham, P. Seller, P. Sellin, R. Speller and M. Veale, "Multiple Module Pixellated CdTe Spectroscopic X-Ray Detector," IEEE Trans. on Nuclear Science, vol. 60, no. 2, pp. 1197-1200, 2013.

[29] M. Veale, A. Schneider, D. Duarte, P. Seller, M. Wilson and S. Bell, "Cadmium Telluride Spectroscopic X-Ray Imaging Detectors," in Position Sensitive Detectors 10, At University of Surrey, Guildford, UK, 2014.

[30] M. Gubarev, B. Ramsey and W. Arnold, in Proc. SPIE 7360, 2009.

[31] M. Gubarev, B. Ramsey, S. O'Dell, R. Elsner, K. Kilaru, J. McCracken, M. Pavlinsky, A. Tkachenko, I. Lapshov, C. Atkins and V. Zavlin, "Development of mirror modules for the ART-XC instrument aboard the Spectrum-Roentgen-Gamma mission," in Proc. SPIE 8861, 88610K, 2013.

[32] S. Krucker, S. Christe, L. Glesener, S. Ishikawa, B. Ramsey, M. Gubarev, S. Saito, T. Takahashi, S.
Watanabe, H. Tajima, T. Tanaka, P. Turin, D. Glaser, J. Fermin and R. Lin, "The focusing optics X-ray solar imager (FOXSI): instrument and first flight," in Proc. SPIE 8862, 88620R, 2013.

[33] K. Kilaru, B. Ramsey, M. Gubarev and D. Gregory, "Differential deposition technique for figure corrections in grazing-incidence x-ray optics," Optical Engineering, vol. 50, no. 10, pp. 106501-106501-6, 2011.

[34] K. Kilaru, D. Merthe, Z. Ali, M. Gubarev, T. Kester, C. Benson, W. McKinney, P. Takacs and V. Yashchuk, "Development of multi-beam long trace profiler," in Proc. SPIE 8147, 814719, 2011.

[35] "WISE MidEX Mission-Press Kit Document," [Online]. Available: http://wise.ssl.berkeley.edu/mission.html. [Accessed 2014].

[36] F. Harrison, F. Christensen, W. Craig, C. Hailey, W. Baumgartner, J. Chonko, W. R. Cook, J. Koglin, K. Madsen, M. Pivavoroff, S. Boggs, D. Smith and C. Chen, "Development of the HEFT and NuSTAR focusing telescopes," in Focusing Telescopes in Nuclear Astrophysics, Springer Netherlands, 2006, pp. 131-137.

[37] "CREAM MIssion Overview - link to ISS-CREAM," 2013. [Online]. Available: http://cosmicray.umd.edu/cream/.

[38] G. J. Fishman, "The History and Legacy of BATSE," in Proceedings of Science: Gamma-Ray Bursts 2012 Conference, Munich, Germany, 2012.

[39] M. Gubarev, B. Ramsey, S. O'Dell, R. Elsner, K. Kilaru, J. McCracken, M. Pavlinsky, A. Tkachenko and I. Lapshov, "The Marshall Space Flight Center development of mirror modules for the ART-XC instrument aboard the Spectrum-Roentgen-Gamma mission," in Proc. SPIE 8443, 84431U, 2012.

[40] C. Atkins, B. Ramsey, K. Kilaru, M. Gubarev, S. O'Dell, R. Elsner, D. Swartz, J. Gaskin and M. Weisskopf, "X-ray optic developments at NASA's MSFC," in Proc. SPIE 8777, 87770W, 2013.

[41] B. D. Ramsey, private communication, based on recent results that are not yet published. The FOXSI-2 optics were delivered in September, 2014 to UC Berkeley.. 


\section{BIOGRAPHY}

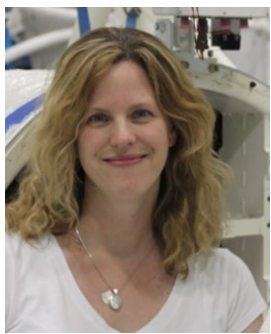

Dr. Jessica Gaskin is a physicist in the X-Ray Astronomy Group at NASA Marshall Space Flight Center. She has worked on multiple types of solid state detector systems for X-Ray Astronomy and has supported the High Energy replicated Optics hard X-ray balloon-borne telescope; offering flight support and detector calibration. Dr. Gaskin is the MSFC PI for HEROES telescope. She has a B.S. in Physics (specializing in Astrophysics) from New Mexico Tech, an M.S. in Astronomy from Case Western Reserve University, and a Ph.D. in Physics from the University of Alabama in Huntsville.

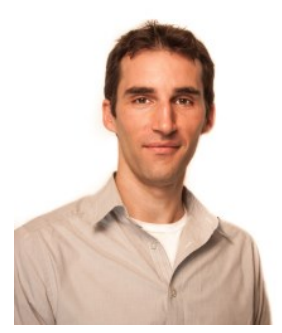

Dr. Steven D. Christe is a Research Astrophysicist at the Solar Physics Laboratory, located at GSFC. Dr. Christe received his Ph.D. from the University of California, Berkeley (UCB), at the Space Sciences Laboratory (SSL) where he developed a sounding rocket program to study solar microflares.

The FOXSI program, short for the Focusing Optics X-ray Solar Imager, is a partnership between the SSL UCB, $M S F C$, and the Japanese Astro-H mission. As a postdoctoral researcher at the SSL, Dr. Christe oversaw the FOXSI program as the project manager/project scientist. These responsibilities have continued after he joined the GSFC in the Fall of 2009. Dr. Christe is the GSFC PI for the HEROES telescope.

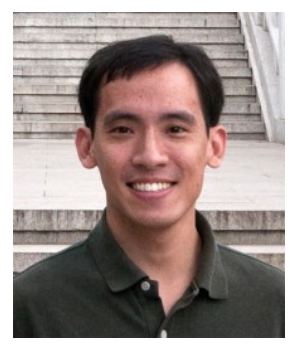

Dr. Albert Shih joined the Solar Physics Laboratory at GSFC in 2010. His research interests focus on $X$-ray and gamma-ray observations of particle acceleration in solar flares. He is currently serving as the Deputy Mission Scientist for RHESSI, and is the project manager and project scientist for Gamma Ray Imager/Polarimeter for Solar flares. Dr. Shih is the Solar Project Scientist for the HEROES payload.

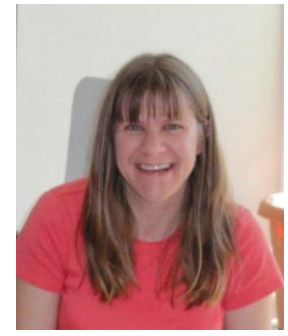

Dr. Colleen Wilson-Hodge began her NASA career as a Cooperative (Co-Op) Education student in 1989, and in 1999 she completed her Ph.D. She has led/co-authored more than 40 refereed journal articles and has led 14 observing proposals. Since 2005, Dr. Wilson-Hodge has been a Co-I on the Gamma-ray Burst Monitor onboard Fermi. She is Astrophysics Project Scientist for HEROES.

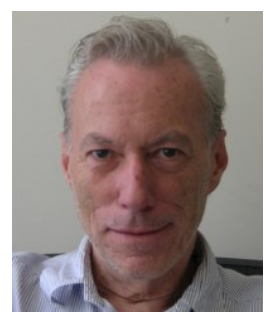

Dr. Brian Ramsey obtained his Ph.D. in Astrophysics from Birmingham University in England, in 1978. Since 1983, he has been at MSFC working in the $x$-ray astronomy group. His areas of interest include $x$-ray and neutron optics and the development of $x$-ray detectors for various applications (including astrophysics, planetary, and medical). Dr. Ramsey was responsible for the conception and implementation of the HERO payload. He serves as an advisor to the HEROES team.

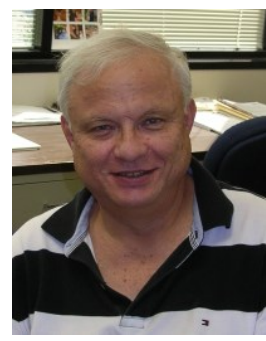

Dr. Ronald F. Elsner obtained his Ph.D. in theoretical astrophysics from the University of Illinois, Urbana-Champaign, in 1976, after 2 years in the U.S. Army. Following a subsequent 2 year postdoc at Cornell University, he has worked in the $x$ ray astronomy group at MSFC since 1978. His recent areas of interest primarily include various aspects of grazing incidence optics, but also x-ray emission from planets and globular clusters.

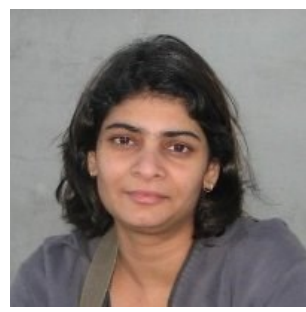

Dr. Kiranmayee Kilaru works with the X-ray astronomy group of NASA Marshall Space Flight Center in Huntsville Al. She supports $X$-ray optics development including the differential deposition technique being developed for figure correction of x-ray mirrors. She has a M.S. degree in physics, with specialization in optics from the National Institute of Technology, Warangal, India, an M.S. degree in Physics and a Ph.D. in Optical Science and Engineering from the University of Alabama in Huntsville.

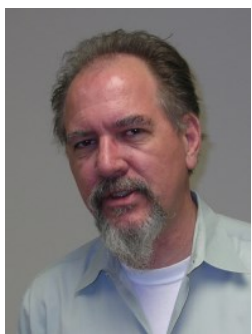

Dr. Douglas Swartz received a Ph.D. in Physics from the University of Texas at Austin in 1989. He has served as a Research Scientist for the Chandra Project Science team, headquartered at MSFC, since 1995. His studies in X-ray astrophysics include Ultraluminous X-ray sources, the growth and evolution of nearby galaxies, and pulsar wind nebulae. He has over 90 publications in refereed scientific journals. Dr. Swartz supports the calibration and data analysis efforts for HEROES.

Dr. Allyn Tennant received his BS in Physics from the University of Texas, Austin and PhD from University of Maryland, College Park. Since 1988 he has worked at Marshall Space Flight Center as AXAF (renamed to 
Chandra) operations scientist. He has been involved in the analysis of data from a variety of missions including HEAO-1, EXOSAT, RXTE, Rosat and Chandra.

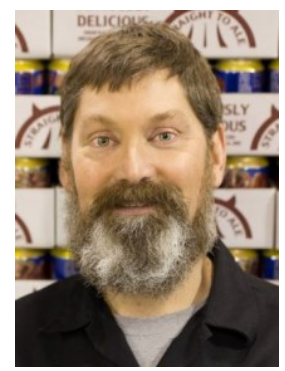

Bruce Weddendorf is a mechanical design engineer specializing in $x$ Ray optical systems design for over 20 years. Bruce has designed instruments, replicated optics fabrication tooling, optical coating systems, and detectors to support $x$ Ray astronomy. He has a B.S. in Mechanical Engineering from the University of South Carolina.

Paul Seller is the Group leader of Detector Development at Rutherford Appleton Laboratory in Oxford UK. The group works on Solid state and Gas detectors for X-ray and particle imaging for many of the large facilities run by the UK STFC. Over the last decade Paul has focussed on CdTe detector technology and advanced ASIC readout of this material. These have been used in synchrotron, medical, industrial inspection and security applications. Paul has a BSc from Queen Mary College London and Is a Chartered Electronics Engineer MIET.

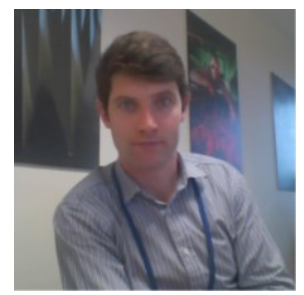

Matt Wilson is a Senior Detector Scientist within the Science and Technology Facilities Council in the UK. Matt has led numerous projects to deliver $X$-ray detector technology to scientific and commercial customers. He has been a key member of the team that have developed the HEXITEC detector technology and supported end users in the operation and integration of the detector. Matt has an MSc in Physics from the University of Nottingham and is a Charted Physicist with the IoP.

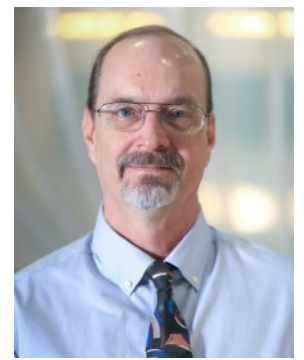

David Stuchlik is an Aerospace Engineer in the Balloon Program Office at NASA's Wallops Flight Facility, which is part of the Goddard Space Flight Center. He has managed several large NASA technology development projects, including the Long Duration Balloon Support Systems, the TDRSS High Gain Antenna for Balloons, and the McMurdo 10 meter Ground Station. Mr. Stuchlik is currently managing the development and application of the Wallops Arc Second Pointer, which has successfully completed five test flights. He has a B.S. in Electronics Engineering from Virginia Tech. 